\title{
WEAK FINSLER STRUTURES AND THE FUNK METRIC
}

\author{
ATHANASE PAPADOPOULOS AND MARC TROYANOV
}

\begin{abstract}
We discuss general notions of metrics and of Finsler structures which we call weak metrics and weak Finsler structures. Any convex domain carries a canonical weak Finsler structure, which we call its tautological weak Finsler structure. We compute distances in the tautological weak Finsler structure of a domain and we show that these are given by the so-called Funk weak metric. We conclude the paper with a discussion of geodesics, of metric balls and of convexity properties of the Funk weak metric.
\end{abstract}

AMS Mathematics Subject Classification: 52A, 53C60, 58B20 Keywords: Finsler structure, weak metric, Funk weak metric.

\section{Contents}

1. Introduction

2. Preliminaries on convex geometry

3. The notion of weak metric

4. Weak length spaces

5. Weak Finsler structures

6. The tautological weak Finsler structure

7. The Funk weak metric

8. On the geometry of the Funk weak metric References

\section{INTRODUCTION}

A weak metric on a set is a function defined on pairs of points in that set which is nonnegative, which can take the value $\infty$, which vanishes when the two points coincide and which satisfies the triangle inequality. Compared to an ordinary metric, a weak metric can thus degenerate and take infinite values. Besides, it need not be symmetric. This is a very general notion which turns out to be useful in various situations. The terminology "weak metric" is due to Ribeiro [20, but the notion can be at least traced back to the work of Hausdorff (see [14]). In the paper [18, a number of natural weak metrics are discussed. In the present paper, we are mostly interested in a class of weak metrics that is related to convex geometry and to a general notion of Finsler structures on manifolds.

A basic construction in convex geometry is the notion of Minkowski norm, which associates to any convex set containing the origin in a vector space $V$ a translationinvariant homogenous weak metric on $V$. Finsler geometry is an extension of this

Date: April 3, 2008. 
construction to an arbitrary manifold. We define a weak Finsler strucure on a differentiable manifold to be a field of convex sets on that manifold. More precisely, a weak Finsler strucure is a subset of the tangent space of the manifold whose intersection with each fiber is an convex set containing the origin. The Minkowski norm in each tangent space of a manifold endowed with a weak Finsler structure gives rise to a function defined on the total space of the tangent bundle. We call this function the Lagrangian of the weak Finsler structure. Integrating this Lagrangian on piecewise smooth curves in the manifold defines a length structure and thus a notion of distance on the manifold. This distance is generally a weak metric.

A case of special interest is when the manifold is a convex domain $\Omega$ in $\mathbb{R}^{n}$ and when the weak Finsler structure is obtained by replicating at each point of $\Omega$ the domain $\Omega$ itself. We call this the tautological weak Finsler structure, and we study some of its basic properties in the present paper. More precisely, we first give a formula for the distance between two points. It turns out that this distance coincides with the metric introduced by P. Funk in [13]. We then study the geometry of balls and the geodesics in the Funk weak metric.

Modern references on Finsler geometry include [9, 2], [3], and [1]. One of Herbert Busemann's major ideas, expressed in [5], 6], 7] and [8] is that Finsler geometry should be developed without local coordinates and without the use of differential calculus. This paper brings some results in that direction.

\section{Preliminaries on CONVEX GeOmetry}

In this section, we recall a few notions in convex geometry that will be used in the sequel.

Given a convex subset $\Omega$ of $\mathbb{R}^{n}$, we shall denote $\bar{\Omega}$ its closure, $\stackrel{o}{\Omega}$ its interior, and $\partial \Omega=\bar{\Omega} \backslash \stackrel{o}{\Omega}$ its boundary.

Let $\Omega \subset \mathbb{R}^{n}$ be a (not necessarily open) convex set and let $x$ be a point in $\Omega$.

Definition 2.1. The radial function of $\Omega$ with respect to $x$ is the function $r_{\Omega, x}$ : $\mathbb{R}^{n} \rightarrow \mathbb{R}_{+} \cup\{\infty\}$ defined by

$$
r_{\Omega, x}(\xi)=\sup \{t \in \mathbb{R} \mid(x+t \xi) \in \Omega\} .
$$

Definition 2.2. The Minkowski function of $\Omega$ with respect to $x$ is the function $p_{\Omega, x}: \mathbb{R}^{n} \rightarrow \mathbb{R}_{+} \cup\{\infty\}$ defined by

$$
p_{\Omega, x}(\xi)=\frac{1}{r_{\Omega, x}(\xi)} .
$$

Classically, the Minkowski function is associated to an open convex subset $\Omega$ of $\mathbb{R}^{n}$ containing the origin 0 , and taking $x=0$. This function is sometimes called the Minkowski weak norm of the convex (see e.g. [10, [17, 21] and [22]).

We also recall that for any convex set $\Omega$ in $\mathbb{R}^{n}$, there exists a well-defined smallest affine subspace $L$ of $\mathbb{R}^{n}$ containing $\Omega$, and that the intersection of $\Omega$ with $L$ has nonempty interior in $L$. We denote by $\operatorname{RelInt}(\Omega)$ this interior, called the relative interior of the convex set $\Omega$.

The following proposition collects a few basic properties of the Minkowski function. In particular, Property (8) tells us that we can reconstruct the relative interior of $\Omega$ from the Minkowski function of $\Omega$ at any point. The proofs are easy. 
Proposition 2.3. Let $\Omega$ be a convex subset of $\mathbb{R}^{n}$. For every $x$ in $\Omega$ and for every $\xi$ and $\eta$ in $\mathbb{R}^{n}$, we have

(1) $p_{\Omega, x}(\xi)=\inf \{t \geq 0 \mid \xi \in t(\Omega-x)\}$. (Here, $\Omega-x$ denotes the Minkowski sum of $\Omega$ and $-x$.)

(2) If the ray $\{x+t \xi \mid t \geq 0\}$ is contained in $\Omega$, then $p_{\Omega, x}(\xi)=0$.

(3) $p_{\Omega, x}(\lambda \xi)=\lambda p_{\Omega, x}(\xi)$ for $\lambda \geq 0$.

(4) $p_{\Omega, x}(\xi+\eta) \leq p_{\Omega, x}(\xi)+p_{\Omega, x}(\eta)$.

(5) The Minkowski function $p_{\Omega, x}$ is convex.

(6) If $x$ is in $\stackrel{o}{\Omega}$, then $p_{\Omega, x}$ is continuous.

(7) If $\Omega$ is closed, then $\Omega=\left\{y \in \mathbb{R}^{n} \mid y=x+\xi, p_{\Omega, x}(\xi) \leq 1\right\}$.

(8) $\operatorname{RelInt}(\Omega)=\left\{y=x+\xi \mid p_{\Omega, x}(\xi)<1\right\}$.

(9) If $\Omega_{1}=\operatorname{RelInt}(\Omega)$, then $p_{\Omega_{1}, x}=p_{\Omega, x}$.

In some cases, we can give explicit formulas for the Minkowski function $p_{\Omega, x}$. For instance, the Minkowski function of the closed ball $B=B(0, R)$ in $\mathbb{R}^{n}$ of radius $R$ and center 0 with respect to any point $x$ in $B$ is given by

$$
p_{B, x}(\xi)=\frac{\sqrt{\langle\xi, x\rangle^{2}+\left(R^{2}-|x|^{2}\right)|\xi|^{2}}+\langle\xi, x\rangle}{\left(R^{2}-|x|^{2}\right)} .
$$

The Minkowski function of a half-space $H=\left\{x \in \mathbb{R}^{n} \mid\langle\nu, x\rangle \leq s\right\}$, where $\nu$ is a vector in $\mathbb{R}^{n}$ (which is orthogonal to the hyperplane bounding $H$ ) and where $s$ is a real number, with respect to a point $x$ in $H$, is given by

$$
p_{H, x}(\xi)=\max \left(\frac{\langle\nu, \xi\rangle}{s-\langle\nu, x\rangle}, 0\right) .
$$

We shall use this formula later on in this paper. We also recall the following:

Definition 2.4 (Support hyperplane). Let $\Omega$ be a nonempty subset of $\mathbb{R}^{n}$. An affine hyperplane $A$ in $\mathbb{R}^{n}$ is called a support hyperplane for $\Omega$ if the relative interior of $\Omega$ is contained in one of the two closed half-spaces bounded by $A$ and if $\bar{\Omega} \cap A \neq \emptyset$. If $A$ is a support hyperplane for $\Omega$ and if $x$ is a point in $\bar{\Omega} \cap A$, then $A$ is called a support hyperplane for $\Omega$ at $x$. When $\Omega \subset \mathbb{R}^{2}$, then $A$ is called a support line.

Suppose now that $\Omega$ a convex subset of $\mathbb{R}^{n}$. It is known that any point on the boundary of $\Omega$ is contained in at least one of its support hyperplanes (see e.g. [10] p. 20). The intersection of $\Omega$ with any of its support hyperplanes is a convex set which is nonempty if $\Omega$ is closed. This intersection is not always reduced to a point. We recall the notion of a strictly convex subset in $\mathbb{R}^{n}$, and before that we note the following classical proposition:

Proposition 2.5. Let $\Omega$ be an open convex subset of $\mathbb{R}^{n}$. Then, the following are equivalent:

(1) $\partial \Omega$ does not contain any nonempty open affine segment;

(2) each support hyperplane of $\Omega$ intersects $\partial \Omega$ in exactly one point;

(3) support hyperplanes at distinct points of $\partial \Omega$ are distinct;

(4) any linear function on $\mathbb{R}^{n}$ has exactly one maximum on $\partial \Omega$.

Definition 2.6 (Strictly convex subset). Let $\Omega$ be an open convex subset of $\mathbb{R}^{n}$. Then, $\Omega$ is said to be strictly convex if one (or, equivalently, all) the properties of Proposition 2.5 are satisfied. 


\section{The NOTION OF WEAK METRIC}

Definition 3.1. A weak metric on a set $X$ is a function $\delta: X \times X \rightarrow \mathbb{R}_{+} \cup\{\infty\}$ satisfying

(1) $\delta(x, x)=0$ for all $x$ in $X$;

(2) $\delta(x, z) \leq \delta(x, y)+\delta(y, z)$ for all $x, y$ and $z$ in $X$.

We say that such a weak metric $\delta$ is symmetric if $\delta(x, y)=\delta(y, x)$ for all $x$ and $y$ in $X$; that it is finite if $\delta(x, y)<\infty$ for every $x$ and $y$ in $X$; that $\delta$ is strongly separating if we have the equivalence

$$
\min (\delta(x, y), \delta(y, x))=0 \Longleftrightarrow x=y ;
$$

and that $\delta$ is weakly separating if we have the equivalence

$$
\max (\delta(x, y), \delta(y, x))=0 \Longleftrightarrow x=y .
$$

We recall that the notion of weak metric already appears in the work of Hausdorff (cf. [14, in which Hausdorff defines asymmetric distances on various sets of subsets of a metric space).

Definition 3.2 (Geodesic). Let $(X, \delta)$ be a weak metric space and let $I \subset \mathbb{R}$ be an interval. We say that a map $\gamma: I \rightarrow X$ is geodesic if for every $t_{1}, t_{2}$ and $t_{3}$ in $I$ satisfying $t_{1} \leq t_{2} \leq t_{3}$ we have

$$
\delta\left(\gamma\left(t_{1}\right), \gamma\left(t_{2}\right)\right)+\delta\left(\gamma\left(t_{2}\right), \gamma\left(t_{3}\right)\right)=\delta\left(\gamma\left(t_{1}\right), \gamma\left(t_{3}\right)\right) .
$$

Weak metrics were extensively studied by Busemann, cf. [5], [6], [7] \& [8]. A basic example of a weak metric defined on a convex set in $\mathbb{R}^{n}$ is the following:

Example 3.3. Let $\Omega \subset \mathbb{R}^{n}$ be a convex set such that $0 \in \bar{\Omega}$ and let $p(\xi)=$ $p_{\Omega, 0}(\xi)=\inf \{t>0 \mid \xi \in t \Omega\}$ be the Minkowski weak norm centered at 0 of $\Omega$. Then, the function $\delta: \mathbb{R}^{n} \times \mathbb{R}^{n} \rightarrow \mathbb{R}_{+} \cup\{\infty\}$ defined by

$$
\delta(x, y)=p(y-x)
$$

is a weak metric on $\mathbb{R}^{n}$. For this weak metric, we have the following equivalences:

(1) $\delta$ is finite $\Longleftrightarrow 0 \in \stackrel{o}{\Omega}$;

(2) $\delta$ is symmetric $\Longleftrightarrow \Omega=-\Omega$;

(3) $\delta$ is strongly separating $\Longleftrightarrow \Omega$ is bounded;

(4) $\delta$ is weakly separating $\Longleftrightarrow \Omega$ does not contain any Euclidean line.

The weak metric on $\mathbb{R}^{n}$ defined in Example 3.3 is called the Minkowski weak metric associated to $\Omega$. The associated weak metric space $\left(\mathbb{R}^{n}, \delta\right)$ is called a weak Minkowski space.

\section{Weak Length spaces}

Let $X$ be a set and let $\Gamma$ be a groupoid of paths in $X$. Concatenation of paths is denoted by the symbol $*$. The inverse $\gamma^{-1}$ of a path $\gamma:[a, b] \rightarrow X$ is the path obtained by pre-composing $\gamma$ with the unique affine sense-reversing self-homeomorphism of $[a, b]$.

Definition 4.1. A weak length structure on $(X, \Gamma)$ is a function $\ell: \Gamma \rightarrow \mathbb{R}_{+} \cup\{\infty\}$ which satisfies the following properties: 
(1) Invariance under reparametrization: if $[a, b]$ and $[c, d]$ are intervals of $\mathbb{R}$, if $\gamma:[a, b] \rightarrow X$ is a path in $X$ that belongs to $\Gamma$ and if $f:[c, d] \rightarrow[a, b]$ is an increasing homeomorphism such that $\gamma \circ f \in \Gamma$, then $\ell(\gamma)=\ell(\gamma \circ f)$.

(2) Additivity: for every $\gamma_{1}$ and $\gamma_{2}$ in $\Gamma$, we have $\ell\left(\gamma_{1} * \gamma_{2}\right)=\ell\left(\gamma_{1}\right)+\ell\left(\gamma_{2}\right)$.

A weak length structure $\Gamma$ is said to be reversible if for every $\gamma$ in $\Gamma, \gamma^{-1}$ is also in $\Gamma$ and we have $\ell\left(\gamma^{-1}\right)=\ell(\gamma)$.

A weak length structure $\Gamma$ is said to be separating if we have the equivalence: $\ell(\gamma)=0 \Longleftrightarrow \gamma$ is a unit in $\Gamma$ (i.e. $\gamma$ is a constant path).

Let $(X, \Gamma, \ell)$ be a set equipped with a groupoid of paths and with a weak length structure. We set

$$
\delta_{\ell}(x, y)=\inf _{\gamma \in \Gamma_{x, y}} \ell(\gamma)
$$

where

$$
\Gamma_{x, y}=\{\gamma \in \Gamma \mid \gamma \text { joins } x \text { to } y\} .
$$

It is easy to see that the function $\delta_{\ell}$ is a weak metric on $X$.

Definition 4.2. Let $(X, \Gamma, \ell)$ be a set equipped with a groupoid of paths and with a weak length structure. The weak metric $\delta_{\ell}$ defined in (4.1) is called the weak metric associated to the weak length structure $\ell$. A weak length metric space is a weak metric space $X$ obtained from such a triple $(X, \Gamma, \ell)$ by equipping $X$ with the associated weak metric $\delta_{\ell}$.

\section{Weak Finsler structures}

We introduce a general notion of Finsler structure, which we call weak Finsler structure, and which can be considered as an infinitesimal notion of weak length structure.

Definition 5.1. Let $M$ be a $C^{1}$ manifold and let $T M$ be its tangent bundle. A weak Finsler structure on $M$ is a subset $\widetilde{\Omega} \subset T M$ such that for each $x$ in $M$, the subset $\Omega_{x}=\widetilde{\Omega} \cap T_{x} M$ of the tangent space $T_{x} M$ of $M$ at $x$ is convex and contains the origin.

We provide the set of all weak Finsler structures on $M$ with the order relation $\preceq$ defined as follows:

$$
\widetilde{\Omega_{1}} \preceq \widetilde{\Omega_{2}} \Leftrightarrow \widetilde{\Omega_{1}} \supset \widetilde{\Omega_{2}} .
$$

Examples 5.2. In the following examples, $M$ is a $C^{1}$ manifold.

(1) $\widetilde{\Omega}=T M$ is a weak Finsler structure, which we call the minimal weak Finsler structure.

(2) $\widetilde{\Omega}=M \subset T M$, embedded as the zero section, is a weak Finsler structure which we call the maximal weak Finsler structure.

(3) If $\widetilde{\Omega}$ and $\widetilde{\Omega^{\prime}}$ are two Finlser structures on $M$, then $\widetilde{\Omega} \cap \widetilde{\Omega^{\prime}} \subset T M$ is also a Finsler structure.

(4) If $\widetilde{\Omega}$ and $\widetilde{\Omega^{\prime}}$ are two Finlser structures on $M$, then, taking the union of the Minkowski sums $\Omega_{x}+\Omega_{x}^{\prime}$ of the convex sets in each tangent space $T_{x} M$, we obtain the Minkowski sum Finsler structure $\widetilde{\Omega}+\widetilde{\Omega^{\prime}} \subset T M$.

(5) If $\omega$ is a differential 1 -form on $M$, then

$$
\widetilde{\Omega}_{\omega}=\left\{(x, \xi) \in T M \mid \omega_{x}(\xi) \leq 1\right\}
$$


and

$$
\widetilde{\Omega}_{|\omega|}=\left\{(x, \xi) \in T M|| \omega_{x} \mid(\xi) \leq 1\right\}
$$

are weak Finsler structures on $M$.

(6) If $\omega$ and $\omega^{\prime}$ are two 1 -froms on $M$, then $\max \left(\omega, \omega^{\prime}\right)$ defines a weak Finlser structure on $M$.

(7) If $\widetilde{\Omega}$ is a weak Finlser structure on $M$ and if $N \subset M$ is a $C^{1}$ submanifold, then $\widetilde{\Omega}_{N}=\widetilde{\Omega} \cap T N$ is a weak Finlser structure on $N$, called the weak Finsler structure induced by the embedding $N \subset M$.

(8) If $\widetilde{\Omega}$ is a weak Finlser structures on $M$, if $N$ is a $C^{1}$ manifold and if $f$ : $N \rightarrow M$ is a $C^{1}$ map, then $(T f)^{-1}(\widetilde{\Omega}) \subset T N$ is a Finsler structure on $N$. We denote it by $f^{*}(\widetilde{\Omega})$ and call it the pull back of $\widetilde{\Omega}$ by the map $f$.

Definition 5.3 (Lagrangian). The Lagrangian of a weak Finlser structure $\widetilde{\Omega}$ on a $C^{1}$ manifold $M$ is the function on the tangent bundle $T M$ whose restriction to each tangent space $T_{x}$ is the Minkowski function of $\Omega_{x}$. It is thus defined by

$$
p(x, \xi)=p_{\widetilde{\Omega}}(x, \xi)=\inf \left\{t \mid t^{-1} \xi \in \Omega_{x}\right\} .
$$

The quantity $p(x, \xi)$ is also called the Finsler norm of the vector $(x, \xi)$ relative to the given weak Finlser structure.

Example 5.4. Let $g$ be a Riemannian metric on $M$, let $\omega$ is a differential 1-form and let $\mu$ be a smooth function on $M$ satisfying $\left|\mu \omega_{x}\right|<1$ at every point $x$ in $M$. Then, $p=\sqrt{g}+\mu \omega$ is the Lagrangian of a Finsler structure on $M$. Such a Finlser structure is usually called a Randers metric on $M$, and it has applications on physics (cf. e.g. 3] $\S 11.3$, and see also [4] for the relation of this metric with the Zermelo navigation problem.)

Lemma 5.5. Let $\widetilde{\Omega}$ be a weak Finlser structure on $M$. Assume that $M$ (considered as a subset of $T M$ - the zero section) is contained in the interior of $\widetilde{\Omega} \subset T M$. Then the associated Lagrangian $p: T M \rightarrow \mathbb{R}$ is upper semi-continuous.

Proof. The hypothesis implies that for every $x$ in $M$, the interior of each convex set $\Omega_{x}=\widetilde{\Omega} \cap T_{x} M \subset T_{x} M$ is nonempty. Therefore, the usual interior and the relative interior of $\Omega_{x}$ coincide. Property (9) of Proposition 2.3 implies then that the Lagrangian of $\widetilde{\Omega}$ coincides with the Lagrangian of its interior $\operatorname{Int}(\widetilde{\Omega})$.

One may therefore assume without loss of generality that $\widetilde{\Omega} \subset T M$ is an open set, and in particular

$$
\widetilde{\Omega}=\{(x, \xi) \in T M \mid p(x, \xi)<1\}
$$

(see Proposition 2.3 (8) ). Now for any $t \in \mathbb{R}$, the sublevel set $\{p(x, \xi)<t\}$ is either empty (when $t \leq 0$ ) or it is homothetic to the open set $\widetilde{\Omega} \subset T M$ (when $t>0$ ). In any case, it is an open subset of $T M$, and $p: T M \rightarrow \mathbb{R}$ is therefore upper semi-continuous.

Proposition 5.6. Let $\widetilde{\Omega}$ be a Finsler structure on a $C^{1}$ manifold $M$ and let $p_{\widetilde{\Omega}}$ : $T M \rightarrow \mathbb{R}$ be the associated Lagrangian. Then,

(1) for every $x$ in $M$, the function $\xi \mapsto p(x, \cdot)$ is a weak norm on $T_{x} M$; 
(2) if $\widetilde{\Omega^{\prime}} \subset T M$ is another Finsler structure on $M$, with associated Lagrangian $p_{\widetilde{\Omega^{\prime}}}$, then we have the equivalence

$$
\widetilde{\Omega} \preceq \widetilde{\Omega^{\prime}} \Longleftrightarrow p_{\widetilde{\Omega}} \leq p_{\widetilde{\Omega^{\prime}}},
$$

(3) $p_{\widetilde{\Omega}}: T M \rightarrow \mathbb{R}$ is Borel-measurable.

Proof. The first two assertions are easy to check and we only prove the last one. If $M$ is contained in the interior of $\widetilde{\Omega} \subset T M$, then, by Lemma 5.5, the Lagrangian $p$ is upper semi-continuous and therefore Borel measurable. In the general case, $M$ is contained in $\widetilde{\Omega}$ but not necessarily in its interior. We consider a decreasing sequence

$$
T M \preceq \widetilde{\Omega}_{1} \preceq \widetilde{\Omega}_{2} \preceq \cdots \preceq \widetilde{\Omega}
$$

of weak Finsler structures such that $M$ is contained in the interior of $\widetilde{\Omega}_{j} \subset T M$ for every $j \in \mathbb{N}$ and

$$
\widetilde{\Omega}=\bigcap_{j=1}^{\infty} \widetilde{\Omega}_{j}
$$

We then have $p_{\widetilde{\Omega}_{1}} \leq p_{\widetilde{\Omega}_{2}} \leq \cdots \leq p_{\widetilde{\Omega}}$ and

$$
p_{\widetilde{\Omega}}=\sup _{j} p_{\widetilde{\Omega}_{j}}=\lim _{j \rightarrow \infty} p_{\widetilde{\Omega}_{j}}
$$

Therefore $p_{\widetilde{\Omega}}$ is the limit of a sequence of Borel measurable functions and is thus Borel measurable.

We shall say that the Finlser structure $\widetilde{\Omega}$ is smooth if $p$ is smooth.

Definition 5.7 (The weak length structure associated to a weak Finsler structure). Let $M$ be a $C^{1}$ manifold equipped with a weak Finlser structure $\widetilde{\Omega}$ with Lagrangian $p$. There is an associated weak length structure on $M$, defined by taking $\Gamma$ to be the groupoid of piecewise $C^{1}$ paths, and defining, for each $\gamma:[a, b] \rightarrow M$ in $\Gamma$,

$$
\ell(\gamma)=\int_{a}^{b} p(\gamma(t), \dot{\gamma}(t)) d t
$$

Remark 5.8. In Equation (5.1), $\gamma$ and $\dot{\gamma}$ are continuous, and since $p$ is Borelmeasurable, the map $t \mapsto p(\gamma(t), \dot{\gamma}(t))$ is nonnegative and measurable. Therefore, the Lebesgue integral is well defined.

\section{The tautological weak Finsler structure}

In this section, $\Omega$ is an open convex subset of $\mathbb{R}^{n}$. We shall use the natural identification $T \Omega \simeq \Omega \times \mathbb{R}^{n}$.

Definition 6.1 (The tautological weak Finsler structure). The tautological weak Finsler structure on $\Omega$ is the weak Finsler structure $\widetilde{\Omega} \subset T \Omega$ defined by

$$
\widetilde{\Omega}=\left\{(x, \xi) \in \Omega \times \mathbb{R}^{n} \mid x \in \Omega \text { and } x+\xi \in \Omega\right\} .
$$

This structure is called "tautological" because the fibre over each point $x$ of $\Omega$ is the set $\Omega$ itself (with the origin at $x$ ).

The proof of next proposition follows easily from the definitions. 
Proposition 6.2. Let $\Omega$ be an open convex subset of $\mathbb{R}^{n}$ equipped with its tautological weak Finsler structure $\widetilde{\Omega}$. Then, for every $x$ in $\Omega$, the Finsler norm of any tangent vector $\xi$ at $x$ is given by $p_{\Omega, x}(\xi)$, where $p_{\Omega, x}$ is the Minkowski function of $\Omega$ with respect to $x$.

Given an open convex subset $\Omega$ of $\mathbb{R}^{n}$, we denote by $d_{\Omega}$ the weak length metric associated to the tautological weak Finsler structure on $\Omega$. This weak metric is thus defined by

$$
d_{\Omega}(x, y)=\inf _{\gamma \in \Gamma_{x, y}} \int_{\gamma} p(\gamma(t), \dot{\gamma}(t)) d t .
$$

where $\Gamma_{x, y}$ is the set of piecewise $C^{1}$ paths joining $x$ to $y$.

Lemma 6.3. Let $\Omega$ and $\Omega^{\prime}$ be two convex open subsets of $\mathbb{R}^{n}$ satisfying $\Omega \subset \Omega^{\prime}$, then $d_{\Omega^{\prime}} \leq d_{\Omega}$.

In the rest of this paper, we shall use the following notations: For $x$ and $y$ in $\mathbb{R}^{n}$, we denote by $|x-y|$ their Euclidean distance. Given two distinct points $x$ and $y$ in $\Omega, R(x, y)$ denote the Euclidean ray starting at $x$ and passing through $y$. In the case where $R(x, y) \not \subset \Omega$ we set $a^{+}=a^{+}(x, y)=R(x, y) \cap \partial \Omega$.

Theorem 6.1. Let $\Omega$ be an open convex subset of $\mathbb{R}^{n}$ equipped with its tautological weak Finsler structure. Then, for every $x$ and $y$ in $\Omega$, the Euclidean segment connecting $x$ and $y$ is of minimal length, and the associated weak metric on $\Omega$ is given by

$$
d_{\Omega}(x, y)= \begin{cases}\log \frac{\left|x-a^{+}\right|}{\left|y-a^{+}\right|} & \text {if } x \neq y \text { and } R(x, y) \not \subset \Omega \\ 0 & \text { otherwise. }\end{cases}
$$

Proof. As before, we let $d_{\Omega}$ denote the weak metric defined by the tautological weak Finsler structure on $\Omega$. We also denote by $\ell(\gamma)$ the length of a path $\gamma$ for the tautological weak Finsler weak length structure.

The proof of the theorem is done in four steps.

Step 1.- Suppose that $R(x, y) \subset \Omega$. Consider the linear path $\gamma:[0,|x-y|] \rightarrow \Omega$ defined by

$$
\gamma(t)=x+t \frac{y-x}{|y-x|} .
$$

The derivative of the path $\gamma$ is the constant vector

$$
\dot{\gamma}(t)=\frac{y-x}{|y-x|} .
$$

Therefore, $p_{\widetilde{\Omega}}(\gamma(t), \dot{\gamma}(t))=\frac{1}{|y-x|} p_{\widetilde{\Omega}}(\gamma(t), y-x)$, which is equal to 0 since $R(x, y) \subset$ $\Omega$.

Now the path $\gamma$ has length zero and satisfies $\gamma(0)=x$ and $\gamma(|y-x|)=y$. Therefore $d_{\Omega}(x, y)=0$.

In the rest of this proof, we suppose that $R(x, y) \not \subset \Omega$. 
Step 2. - We show that for every distinct points $x$ and $y$ in $\Omega$ and for every Euclidean segment $\gamma$ joining $x$ to $y$, we have

$$
d_{\Omega}(x, y) \leq \ell(\gamma)=\log \frac{\left|x-a^{+}\right|}{\left|y-a^{+}\right|} .
$$

Using the radial function $r_{\Omega, x}$ introduced in 92 we can write

$$
a^{+}=a(x, y-x)=x+r_{\Omega, x}(y-x) \cdot(y-x) .
$$

To compute the Finsler length of the Euclidean segment $[x, y]$, we parametrize it as the path $\gamma$ defined in (6.2).

For $0 \leq t \leq|x-y|$, let $r(t)=|x-\gamma(t)|$. Then, $r(t)=r_{\Omega, x}(\gamma(t), \dot{\gamma}(t))$, and it is easy to see that

$$
r(t)=\left|x-a^{+}\right|-t .
$$

Then, we have $r^{\prime}(t)=-1$ and therefore

$$
\ell(\gamma)=\int_{0}^{|y-x|} \frac{d t}{r(t)}=-\int_{0}^{|y-x|} \frac{r^{\prime}(t) d t}{r(t)}=-\left.\log (r(t))\right|_{t=0} ^{t=|y-x|}=\log \frac{\left|x-a^{+}\right|}{\left|y-a^{+}\right|} .
$$

This gives the desired inequality (6.3).

Step 3.- We complete the proof of the theorem in the particular case where $\Omega$ is a half-space. By the invariance of the tautological Finsler structure under the group of affine transformations, it suffices to consider the case where $\Omega$ is the half-space $H \subset \mathbb{R}^{n}$ defined by the equation

$$
H=\left\{x \in \mathbb{R}^{n} \mid\langle\nu, x\rangle \leq s\right\},
$$

for some vector $\nu$ in $\mathbb{R}^{n}$ (which is orthogonal to the hyperplane bounding $H$ ) and for some $s$ in $\mathbb{R}$. Recall that the Minkowski function associated to $H$ is given by the formula

$$
p_{H}(x, \xi)=\max \left\{\frac{\langle\nu, \xi\rangle}{s-\langle\nu, x\rangle}, 0\right\} .
$$

Consider now an arbitrary piecewise $C^{1}$ path $\alpha:[0,1] \rightarrow H$ such that $x=\alpha(0)$ and $y=\alpha(1)$. Then,

$$
\ell(\alpha)=\int_{0}^{1} \max \left\{\frac{\langle\nu, \dot{\alpha}(t)\rangle}{s-\langle\nu, \alpha(t)\rangle}, 0\right\} d t \geq \int_{0}^{1} \frac{\langle\nu, \dot{\alpha}(t)\rangle}{s-\langle\nu, \alpha(t)\rangle} d t .
$$

We have

$$
\frac{\langle\nu, \dot{\alpha}(t)\rangle}{s-\langle\nu, \alpha(t)\rangle}=-\frac{d}{d t}(\log (s-\langle\nu, \alpha(t)\rangle)) \text {. }
$$

Therefore,

$$
\ell(\alpha) \geq-\log (s-\langle\nu, \alpha(1)\rangle)+\log (s-\langle\nu, \alpha(0)\rangle)=\log \frac{s-\langle\nu, x\rangle}{s-\langle\nu, y\rangle} .
$$

Now we note that

$$
s-\langle\nu, x\rangle=s-\left\langle\nu, x-a^{+}\right\rangle-\left\langle\nu, a^{+}\right\rangle=\left\langle x-a^{+},-\nu\right\rangle=\left\langle\nu, a^{+}-x\right\rangle .
$$

Likewise,

Thus, we obtain

$$
s-\langle\nu, y\rangle=\langle\nu, a-y\rangle
$$

$$
\ell(\alpha) \geq \log \frac{\left\langle\nu, a^{+}-x\right\rangle}{\left\langle\nu, a^{+}-y\right\rangle}
$$


Now using the fact that the three points $x, y, a^{+}$are aligned in that order and that $\nu$ is not parallel to the vector $x-y$, we easily see that

$$
\frac{\langle\nu, a-x\rangle}{\langle\nu, a-y\rangle}=\frac{\left|x-a^{+}\right|}{\left|y-a^{+}\right|}
$$

which gives

$$
\ell(\alpha) \geq \log \frac{\left|x-a^{+}\right|}{\left|y-a^{+}\right|}
$$

Since $\alpha$ was arbitrary, we have

$$
d_{H}(x, y) \geq \log \frac{\left|x-a^{+}\right|}{\left|y-a^{+}\right|} .
$$

Combining this inequality and the inequality (6.3), we obtain, in the case where $\Omega=H$ is a half-space,

$$
d_{H}(x, y)=\log \frac{\left|x-a^{+}\right|}{\left|y-a^{+}\right|} .
$$

In particular any Euclidean segment is length minimizing.

Step 4.- Now we prove the proposition for a general open convex set $\Omega$.

Let $x$ and $y$ be two elements in $\Omega$ and consider the Euclidean ray $R(x, y)$.

By hypothesis, we have $L \not \subset \Omega$, and as before, we set $a^{+}=R(x, y) \cap \partial \Omega$. We let $A$ denote a support hyperplane to $\Omega$ through $a^{+}$, and we let $H$ be the open half-space containing $\Omega$ and whose boundary is equal to $A$. Using Lemma 6.3 and Step 3, we have

$$
d_{\Omega}(x, y) \geq d_{H}(x, y)=\log \frac{\left|x-a^{+}\right|}{\left|y-a^{+}\right|} .
$$

Combining this with the inequality (6.3) we obtain $d_{\Omega}(x, y)=\log \frac{\left|x-a^{+}\right|}{\left|y-a^{+}\right|}$. The argument also proves that any Euclidean segment $\gamma$ is length minimizing. This completes the proof of Theorem 6.1.

\section{The Funk WeAK Metric}

In this and the following section, we give a quick overview of the Funk weak metric, of its geodesics, of its balls and of its topology.

The Funk weak metric is a nice example of a weak metric, and a geometric study of this weak metric is something which seems missing in the literature. We study this weak metric in more detail in [19].

In this section, $\Omega$ is a nonempty open convex subset of $\mathbb{R}^{n}$. We use the notations $a^{+}, R(x, y)$, etc. established in the preceding section.

Definition 7.1 (The Funk weak metric). The Funk weak metric of $\Omega$, denoted by $F_{\Omega}$, is defined, for $x$ and $y$ in $\Omega$, by the formula

$$
F_{\Omega}(x, y)= \begin{cases}\log \frac{\left|x-a^{+}\right|}{\left|y-a^{+}\right|} & \text {if } x \neq y \text { and } R(x, y) \not \subset \Omega \\ 0 & \text { otherwise. }\end{cases}
$$


Observe that Theorem 6.1 says that the Funk weak metric is the weak metric associated to the tautological Finsler structure in $\Omega$. In particular the triangle inequality is verified. Another proof of the triangle inequality is given in 23] p. 85 . This proof is not trivial and uses arguments similar to those of the classical proof of the triangle inequality for the Hilbert metric, as given by D. Hilbert in [16]. If $\Omega=\mathbb{R}^{n}$, then $F \equiv 0$. We shall henceforth assume that $\Omega \neq \mathbb{R}^{n}$ whenever we shall deal with the Funk weak metric of an nonempty open convex subset $\Omega$ of $\mathbb{R}^{n}$. The Funk weak metric is always unbounded. Indeed, if $x$ is any point in $\Omega$ and if $x_{n}$ is any sequence of points in that space converging to a point on $\partial \Omega$ (convergence here is with respect to the Euclidean metric), then $F_{\Omega}\left(x, x_{n}\right) \rightarrow \infty$. Notice that on the other hand $F_{\Omega}\left(x_{n}, x\right)$ is bounded.

Example 7.2 (The upper half-plane). Let $\Omega=H \subset \mathbb{R}^{2}$ be the upper half-plane, that is,

$$
H=\left\{\left(x_{1}, x_{2}\right) \in \mathbb{R}^{2} \mid x_{2}>0\right\} .
$$

Then, for $x=\left(x_{1}, x_{2}\right)$ and $y=\left(y_{1}, y_{2}\right)$ in $H$, we have

$$
F_{H}(x, y)=\max \left\{\log \frac{x_{2}}{y_{2}}, 0\right\} \text {. }
$$

The following three propositions are easy consequences of the definitions and they will be used below. We take $\Omega$ to be again a nonempty open subset of $\mathbb{R}^{n}$.

Proposition 7.3. Let $\Omega^{\prime} \subset \Omega$ be the intersection of $\Omega$ with an affine subspace of $\mathbb{R}^{n}$, and suppose that $\Omega^{\prime} \neq \emptyset$. Then, $F_{\Omega^{\prime}}$ is the weak metric induced by $F_{\Omega}$ on $\Omega^{\prime}$.

Proposition 7.4. In the case where $\Omega$ is bounded, the Funk weak metric $F_{\Omega}$ is strongly separating, and we have the following equivalences:

$$
F_{\Omega}\left(x, x_{n}\right) \rightarrow 0 \Longleftrightarrow F_{\Omega}\left(x_{n}, x\right) \rightarrow 0 \Longleftrightarrow\left|x-x_{n}\right| \rightarrow 0 .
$$

Proposition 7.5. Let $\Omega_{1}$ and $\Omega_{2}$ be two open convex subsets of $\mathbb{R}^{n}$. Then,

$$
F_{\Omega_{1} \cap \Omega_{2}}=\max \left\{F_{\Omega_{1}}, F_{\Omega_{1}}\right\} .
$$

\section{On the GeOMetry of the Funk WeAK Metric}

In this section, we study the geodesics, and then, the geometric balls of the Funk weak metric.

Proposition 8.1. Let $x, y$ and $z$ be three points in $\Omega$ lying in that order on a Euclidean line. Then, we have $F(x, y)+F(y, z)=F(x, z)$.

This results follows from Theorem 6.1, but it is also quite simple to prove it directly.

Proof. We can assume that the three points are distinct, otherwise the proof is trivial. We have $R(x, y) \subset \Omega \Longleftrightarrow R(x, z) \subset \Omega \Longleftrightarrow R(y, z) \subset \Omega$, and this holds if and only if the three quantities $F(x, y), F(y, z)$ and $F(x, z)$ are equal to 0 . Thus, the conclusion also holds trivially in this case. Therefore, we can assume that $R(x, y) \not \subset \Omega$. In this case, we have $a^{+}(x, y)=a^{+}(x, z)=a^{+}(y, z)$. Denoting this common point by $a^{+}$, we have

$$
\frac{\left|x-a^{+}\right|}{\left|y-a^{+}\right|} \frac{\left|y-a^{+}\right|}{\left|z-a^{+}\right|}=\frac{\left|x-a^{+}\right|}{\left|z-a^{+}\right|}
$$


which implies

$$
\log \frac{\left|x-a^{+}\right|}{\left|y-a^{+}\right|}+\log \frac{\left|y-a^{+}\right|}{\left|z-a^{+}\right|}=\log \frac{\left|x-a^{+}\right|}{\left|z-a^{+}\right|},
$$

which completes the proof.

Corollary 8.2. The Euclidean segments in $\Omega$ are geodesic segments for the Funk weak metric on $\Omega$.

Since the open set $\Omega$ is convex, Corollary 8.2 implies that $\left(\Omega, F_{\Omega}\right)$ is a geodesic weak metric space (any two points can be joined by a geodesic segment). It also says that $\left(\Omega, F_{\Omega}\right)$ is a Desarguesian space in the sense of H. Busemann (see [7]).

Notice that in general, the Euclidean segments are not the only geodesic segments for a Funk weak metric. In fact, the following proposition implies that there exist other types of geodesic segments in $\Omega$, provided there exists a Euclidean segment of nonempty interior contained in the boundary of $\Omega$.

Proposition 8.3. Let $\Omega$ be an open convex subset of $\mathbb{R}^{n}$ such that $\partial \Omega$ contains a Euclidean segment $[p, q]$ and let $x$ and $z$ be two points in $\Omega$ such that $R(x, z) \cap$ $[p, q] \neq \emptyset$. Let $\Omega^{\prime}$ be the intersection of $\Omega$ with the affine subspace of $\mathbb{R}^{n}$ spanned by $\{x\} \cup[p, q]$. Then, for any point $y$ in $\Omega^{\prime}$ satisfying $R(x, y) \cap[p, q] \neq \emptyset$ and $R(y, z) \cap[p, q] \neq \emptyset$, we have $F(x, y)+F(y, z)=F(x, z)$.

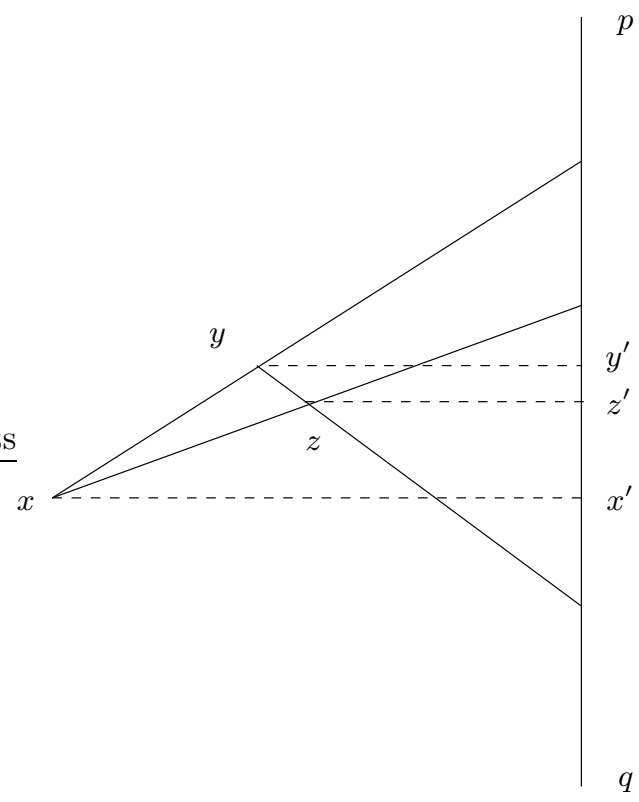

FIGURE 1.

Proof. It suffices to work in the space $\Omega^{\prime}$. Let $x^{\prime}, y^{\prime}$ and $z^{\prime}$ denote the feet of the perpendiculars from $x$ and $z$ respectively on the Euclidean line joining the points $p$ and $q$ (see Figure 1). Let $b=R(x, z) \cap[p, q]$. Since the triangles $b x x^{\prime}$ and $b z z^{\prime}$ are similar, we have

$$
F(x, z)=\log \frac{|x-b|}{|z-b|}=\log \frac{\left|x-x^{\prime}\right|}{\left|z-z^{\prime}\right|} .
$$


Similar formulas hold for $F(x, y)$ and $F(y, z)$. Therefore,

$$
\begin{aligned}
F(x, z) & =\log \frac{\left|x-x^{\prime}\right|}{\left|z-z^{\prime}\right|} \\
& =\log \left(\frac{\left|x-x^{\prime}\right|}{\left|y-y^{\prime}\right|} \frac{\left|y-y^{\prime}\right|}{\left|z-z^{\prime}\right|}\right) \\
& =\log \left(\frac{\left|x-x^{\prime}\right|}{\left|y-y^{\prime}\right|}\right)+\log \left(\frac{\left|y-y^{\prime}\right|}{\left|z-z^{\prime}\right|}\right) \\
& =F(x, y)+F(y, z) .
\end{aligned}
$$

Remark 8.4. By taking limits of polygonal paths, we can easily construct, from Proposition 8.3. smooth paths which are not Euclidean paths and which are geodesic for the Funk weak metric.

Proposition 8.5. Let $\Omega$ be an open convex subset of $\mathbb{R}^{n}$. Let $x$ and $z$ be two distinct points in $\Omega$ such that $R(x, z) \cap \partial \Omega \neq \emptyset$ and such that at the point $b=R(x, z) \cap \partial \Omega$, there is a support hyperplane whose intersection with $\partial \Omega$ is reduced to b. Let $y$ be a point in $\Omega$ such that the three points $x, y, z$ in $\Omega$ do not lie on the same affine line. Then, $F(x, z)<F(x, y)+F(y, z)$.

Proof. To prove the proposition, we work in the affine plane spanned by $x, y$ and $z$ and therefore we can assume without loss of generality that $n=2$.

We assume that the intersection points of $R(x, y)$ and $R(y, z)$ with $\partial \Omega$ are not empty, and we let $a$ and $c$ be respectively these points. From the hypothesis, there is a support line of $\Omega$ (which we call $D$ ) at $b$ whose intersection with $\partial \Omega$ is reduced to the point $b$.

For the proof, we distinguish three cases.

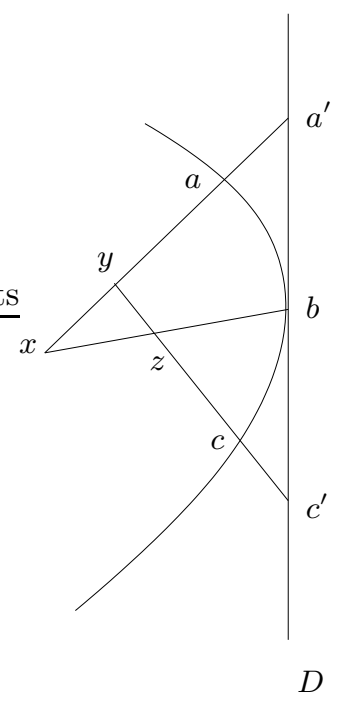

FIGURE 2. 
Case 1.- The two rays $R(x, y)$ and $R(y, z)$ intersect the line $D$ (see Figure 2).

Let $a^{\prime}$ and $c^{\prime}$ be respectively these intersection points. Note that the three points $a^{\prime}, b$ and $c^{\prime}$ are in that order on $D$. By reasoning with projections on the line $D$ and arguing as we did in the proof of Proposition 8.3 we have

$$
\frac{|x-b|}{|z-b|}=\frac{\left|x-a^{\prime}\right|}{\left|y-a^{\prime}\right|} \frac{\left|y-c^{\prime}\right|}{\left|z-c^{\prime}\right|} \text {. }
$$

Since we have

$$
\frac{\left|x-a^{\prime}\right|}{\left|y-a^{\prime}\right|}<\frac{|x-a|}{|y-a|}
$$

and

we obtain

$$
\frac{\left|y-c^{\prime}\right|}{\left|z-c^{\prime}\right|}<\frac{|y-c|}{|z-c|}
$$

$$
\frac{|x-b|}{|z-b|}<\frac{|x-a|}{|y-a|} \frac{|y-c|}{|z-c|}
$$

which gives, by taking logarithms, $F(x, z)<F(x, y)+F(y, z)$.

Case 2.- The ray $R(x, y)$ intersects $D$ and the ray $R(y, z)$ does not intersect $D$ (Figure 3). We let as before $a^{\prime}$ denote the point $R(x, y) \cap D$.

Let $D^{\prime}$ be the Euclidean line passing through $z$ and parallel to $D$. The hypotheses in the case considered imply that the line $D^{\prime}$ intersects the segment $[x, y]$. Let $y^{\prime}$ be this intersection point. The point $y^{\prime}$ is contained in $\Omega$.

We have, as in Case 1,

$$
F(x, z)=\log \frac{|x-b|}{|z-b|}
$$

and

$$
F(x, y)=\log \frac{|x-a|}{|y-a|}>\log \frac{\left|x-a^{\prime}\right|}{\left|y-a^{\prime}\right|} .
$$

Now we have

$$
\frac{|x-b|}{|z-b|}=\frac{\left|x-a^{\prime}\right|}{\left|y^{\prime}-a^{\prime}\right|}<\frac{\left|x-a^{\prime}\right|}{\left|y-a^{\prime}\right|}
$$

that is, $F(x, z)<F(x, y)$, which implies the desired result.

Case 3.- The ray $R(x, y)$ does not intersect the line $D$. This case can be treated as Case 2, and we have in this case $F(x, y)<F(y, z)$, which implies the desired result.

The following is a direct consequence of Proposition 8.5

Corollary 8.6. Let $\Omega$ be an open bounded strictly convex subset of $\mathbb{R}^{n}$ and let $x$, $y$ and $z$ be three points in $\Omega$ that are not contained in an affine segment. Then, $F(x, z)<F(x, y)+F(y, z)$.

Corollary 8.7. Let $\Omega$ be an open bounded strictly convex subset of $\mathbb{R}^{n}$. Then, the affine segments in $\Omega$ are the only geodesic segments for the Funk weak metric of $\Omega$.

Proof. This follows from the previous Corollary and Corollary 8.2 which says that the affine segments are geodesic segments for the Funk weak metric. 


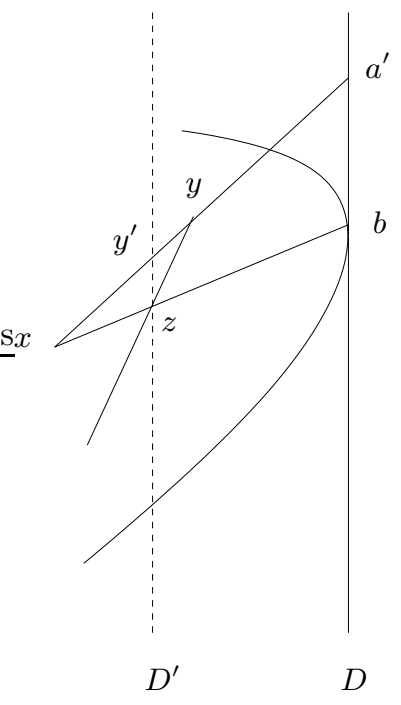

FiguRE 3 .

We recall that a subset $Y$ in a (weak) metric space $X$ is said to be geodesically convex if for any two points $x$ and $y$ in $Y$, any geodesic segment in $X$ joining $x$ and $y$ is contained in $Y$.

Corollary 8.8. Let $\Omega$ be an open bounded strictly convex subset of $\mathbb{R}^{n}$ and let $\Omega^{\prime}$ be a subset of $\Omega$. Then, $\Omega^{\prime}$ is convex with respect to the affine structure of $\mathbb{R}^{n}$ if and only if $\Omega^{\prime}$ is a geodesically convex subset of $\Omega$ with respect to the Funk metric $F_{\Omega}$.

Remark 8.9. Note the formal analogy between Corollary 8.7 and the following well known result on the geodesic segments of a Minkowski metric on $\mathbb{R}^{n}$ : if the unit ball of a Minkowski metric is strictly convex, then the only geodesic segments of this metric are the affine segments.

We now consider spheres and balls in a Funk weak metric space $(\Omega, F)$. As this weak metric is non-symmetric, we have to distinguish between right and left spheres, and we use the following notations. For any point $x$ in $\Omega$ and any nonnegative real number $\delta$, we set

○ $B(x, \delta)=\left\{y \in \Omega \mid F_{\Omega}(x, y)<\delta\right\}$ (the right open ball of center $x$ and radius $\delta$ );

$\circ B^{\prime}(x, \delta)=\left\{y \in \Omega \mid F_{\Omega}(y, x)<\delta\right\}$ (the left open ball of center $x$ and radius $\delta$ );

○ $S(x, \delta)=\left\{y \in \Omega \mid F_{\Omega}(x, y)=\delta\right\}$ (the right sphere of center $x$ and radius $\delta$ );

○ $S^{\prime}(x, \delta)=\left\{y \in \Omega \mid F_{\Omega}(y, x)=\delta\right\}$ (the left sphere of center $x$ and radius $\delta$ ).

In 6] p. 20, H. Busemann discusses topologies for general weak metric spaces. In the case of a genuine metric space, the open balls are used to define the topology of that space. In general, the collections of left and of right open balls in a weak metric space generate two different topologies. For the Funk weak metric, we have the following

If $\Omega$ is a bounded convex open set of $\mathbb{R}^{n}$ equipped with its Funk weak metric; then, the collections of left and of right open balls are sub-bases of the same topology on 
$\Omega$, and this topology coincides with the topology induced from the inclusion of $\Omega$ in $\mathbb{R}^{n}$.

In the case where the convex open set $\Omega$ is unbounded, the left and the right open balls of the Funk weak metric are always noncompact. In the next proposition, we study these balls in the case where $\Omega$ is bounded. We recall that a convex subset of $\mathbb{R}^{n}$ is unbounded if and only if it contains a Euclidean ray.

Proposition 8.10. Let $\Omega$ be a bounded convex open subset of $\mathbb{R}^{n}$, let $x$ be a point in $\Omega$ and let $\delta$ be a nonnegative real number. Then,

(1) The right sphere $S(x, \delta)$ is convex as a subset of $\mathbb{R}^{n}$, and it is compact. Furthermore, this sphere is the image of $\partial \Omega$ by the Euclidean homothety $\sigma$ of center $x$ and factor $\left(1-e^{-\delta}\right)$.

(2) The left sphere $S^{\prime}(x, \delta)$ is convex as a subset of $\mathbb{R}^{n}$, and it is equal to the intersection with $\Omega$ of the image of $\partial \Omega$ by the Euclidean homothety of center $x$ and of factor $\left(e^{\delta}-1\right)$, followed by the Euclidean central symmetry of center $x$. The sphere $S^{\prime}(x, \delta)$ is not necessarily compact.

Proof. Let $y$ be a point in $\Omega$ and let us set, as before, $a^{+}=R(x, y) \cap \partial \Omega$. We have the following equivalences:

$$
y \in S(x, \delta) \Longleftrightarrow \log \frac{\left|x-a^{+}\right|}{\left|y-a^{+}\right|}=\delta \Longleftrightarrow \frac{\left|x-a^{+}\right|}{\left|y-a^{+}\right|}=e^{\delta},
$$

which is easily seen to be equivalent to $|y-x|=\left|x-a^{+}\right|\left(1-e^{-\delta}\right)$. From this fact Property (1) follows easily.

To prove Property (2), let $a^{-}=R(y, x) \cap \partial \Omega$. We have the following equivalences:

$$
\log \frac{\left|y-a^{-}\right|}{\left|x-a^{-}\right|}=\delta \Longleftrightarrow\left|y-a^{-}\right|=e^{\delta}\left|x-a^{-}\right|,
$$

which is also equivalent to

$$
|y-x|=\left(e^{\delta}-1\right)\left|x-a^{-}\right| .
$$

Thus, $y \in S^{\prime}(x, \delta)$ if and only if $y$ is in the intersection of $\Omega$ with the image $\sigma(\partial \Omega)$ of $\partial \Omega$ by the Euclidean homothety with center $x$ and of factor $\left(e^{\delta}-1\right)$, followed by the Euclidean central symmetry of center $x$. This intersection is convex as a subset of $\mathbb{R}^{n}$ but it is not necessarily a compact subset of $(\Omega, F)$. Thus, $S^{\prime}(x, \delta)$ is compact if and only if $\sigma(\partial \Omega)$ is contained in $\Omega$.

We note the following "local-implies-global" property of Funk weak metrics. The meaning of the statement is clear, and it follows directly from Proposition 8.10 (1).

Corollary 8.11. We can reconstruct the boundary $\partial \Omega$ of $\Omega$ from the local geometry at any point of $\Omega$.

Corollary 8.12. Let $\Omega$ be a bounded open strictly convex subset of $\mathbb{R}^{n}$. Then, the left and right open balls of $\Omega$ are geodesically convex with respect to the Funk weak metric $F_{\Omega}$.

Proof. This follows from Proposition 8.10 and from Corollary 8.8 .

We also deduce from Proposition 8.10 that for any $x$ and $x^{\prime}$ in $\Omega$ and for any two positive real numbers $\delta$ and $\delta^{\prime}$, the right spheres $S(x, \delta)$ and $S\left(x^{\prime}, \delta^{\prime}\right)$ are homothetic. 
Thus, for instance, if $\Omega$ is the interior of a Euclidean sphere (respectively, of a Euclidean ellipsoid) in $\mathbb{R}^{n}$, then any right sphere $S(x, \delta)$ is a Euclidean sphere (respectively, an ellipsoid).

Note that the proof of Proposition 8.10 shows that for a fixed $x$, any two right spheres $S(x, \delta)$ and $S\left(x, \delta^{\prime}\right)$ are homothetic by a Euclidean homothety of center $x$, but that in general, a homothety which sends a sphere $S(x, \delta)$ to a sphere $S\left(x^{\prime}, \delta^{\prime}\right)$ does not necessarily send the center $x$ of $S(x, \delta)$ to the center $x^{\prime}$ of $S\left(x^{\prime}, \delta^{\prime}\right)$. One can see this fact on the following example: Let $\Omega$ be an open Euclidean disk in $\mathbb{R}^{n}$, and let us take $x$ to be the Euclidean center of that disk. Then, by symmetry, for any $\delta>0$, the right sphere $S(x, \delta)$ is a Euclidean sphere whose Euclidean and whose metric centers are both at $x$. Now let $x^{\prime}$ be a point which is close to the boundary of $\Omega$. Obviously, the Euclidean homothety that sends $\partial \Omega$ to $S\left(x^{\prime}, \delta\right)$ does not send the center of $\partial \Omega$ to the (Funk-)geometric center of the sphere $S\left(x^{\prime}, \delta\right)$ (recall that the center of this homothety is the point $x$ ). Now taking a composition of two homotheties, we obtain a Euclidean homothety that sends the geometric sphere $S(x, \delta)$ to the geometric sphere $S\left(x^{\prime}, \delta\right)$, and that does not preserve the geometric centers of these spheres.

Remark 8.13. The property for a weak metric on a subset $\Omega$ of $\mathbb{R}^{n}$ that all the right spheres are homothetic is also shared by the metrics induced by Minkowski weak metrics on $\mathbb{R}^{n}$.

\section{REFERENCES}

[1] J. C. Álvarez Paiva \& C. Durán, An introduction fo Finsler geometry, Publicaciones de la Escuela Venezolana de Matematicas (1998).

[2] D. Bao, R. L. Bryant, S. S. Chern \& Z. Shen (editors), A sampler of Finsler geometry, MSRI Publications 50, Cambridge University Press, 2004.

[3] D. Bao, S. S. Chern \& Z. Shen, An introduction to Riemann-Finsler geometry, Graduate Texts in Mathematics, Springer Verlag, 2000.

[4] D. Bao, C. Robles \& Z. Shen, Zermelo navigation on Riemannian manifolds, Journal of Differential Geometry 66 (2004) 377-435.

[5] H. Busemann, Metric methods in Finsler spaces and in the foundations of geometry, Annals of Mathematics Studies 8, Princeton University Press (1942).

[6] H. Busemann, Local metric geometry, Trans. Amer. Math. Soc. 56, (1944) 200-274.

[7] H. Busemann, The geometry of geodesics, Academic Press (1955), reprinted by Dover in 2005.

[8] H. Busemann, Recent synthetic differential geometry, Ergebnisse der Mathematik und ihrer Grenzgebiete, 54, Springer-Verlag, 1970.

[9] S. S. Chern \& Z. Shen, Riemann-Finsler geometry, Nankai Tracts in Mathematics Series, World Scientific Publishing Company, 2005.

[10] H. G. Eggleston, Convexity, Cambridge Tracts in Mathematics and Mathematical Physics No. 47, Cambridge University Press, 1958.

[11] W. Fenchel, Convex cones, sets, and functions, Mimeographed Notes by D. W. Blackett of Lectures at Princeton University, Spring Term, 1951, Princeton, 1953.

[12] P. Finsler, Ueber Kurven und Flchen in allgemeinen Rumen, Göttingen (1918) (Dissertation).

[13] P. Funk, Über Geometrien, bei denen die Geraden die Kürzesten sind, Math. Ann. 101 (1929), 226-237.

[14] F. Hausdorff, Set theory, Chelsea 1957.

[15] D. Hilbert, Ueber die gerade Linie als kürzestes Verbindung zweier Punkte (On the straight line as shortest Connection between two points), Math. Ann. XLVI. 91-96 (1895).

[16] D. Hilbert, Grundlagen der Geometrie, B. G. Teubner, Stuttgart 1899, several later editions revised by the author, and several translations.

[17] H. Minkowski, Theorie der konvexen Körper, insbesondere Begründung ihres Oberflächenbegriffs, in Gesammelte Abhandlungen, Teubner, Leipzig, 1911. 
[18] A. Papadopoulos \& M. Troyanov, Weak metrics on Euclidean domains, JP Journal of Geometry and Topology, Volume 7, Issue 1 (March 2007), pp. 23-44.

[19] A. Papadopoulos \& M. Troyanov, Harmonic symmetrization of convex sets and Hilbert geometry, in preparartion.

[20] H. Ribeiro, Sur les espaces à métrique faible, Portugaliae Math., 4 (1943) 21-40.

[21] A.C. Thompson, Minkowski geometry. Encyclopedia of Mathematics and its Applications, 63. Cambridge University Press, Cambridge, 1996

[22] R. Webster, Convexity, Oxford University Press, 1994.

[23] E. M. Zaustinsky, Spaces with nonsymmetric distance, Mem. Amer. Math. Soc. No. 34, 1959.

A. Papadopoulos, Institut de Recherche Mathématique Avancée, Université louis Pasteur and CNRS, 7 Rue René Descartes, 67084 Strasbourg Cedex - France

E-mail address: papadopoulos@math.u-strasbg.fr

M. Troyanov, Section de Mathématiques, École Polytechnique Fédérale de Lausanne, 1015 LAUSANNE - SWITZERLAND

E-mail address: marc.troyanov@epfl.ch 